\title{
Endoplasmic Reticulum Export Site Formation and Function in Dendrites
}

\author{
Meir Aridor, ${ }^{1}$ Amy K. Guzik, ${ }^{2}$ Anna Bielli, ${ }^{1}$ and Kenneth N. Fish ${ }^{2}$ \\ ${ }^{1}$ Department of Cell Biology and Physiology, University of Pittsburgh School of Medicine, Pittsburgh, Pennsylvania 15261, and ${ }^{2}$ The Harold L. Dorris \\ Neurological Research Center and Department of Neuropharmacology, The Scripps Research Institute, La Jolla, California 92037
}

\begin{abstract}
The elongated and polarized characteristics of neurons render targeting of receptors to the plasma membrane of distal axonal projections and dendritic branches a major sorting task. Although the majority of biosynthetic cargo synthesis, transport, and sorting are believed to occur in the soma, local membrane protein translation and sorting has been reported recently to take place in dendrites and axons. We investigated where endoplasmic reticulum (ER) export occurs in dendrites using an in vitro permeabilized neuron system that enables us to specifically control the assembly of ER export sites. We show that ER export sites are assembled regularly throughout the entire dendritic tree by the regulated sequential recruitment of Sarl and COPII (coat protein complex II). Moreover, activation of metabotropic glutamate receptors leads to the recruitment of the NMDA receptor subunit NR1 to remodeled ER export sites. We propose that regulation of receptor assembly and export from the ER in dendrites plays an important role in modulating receptor surface expression and neuronal function.
\end{abstract}

Key words: Sar1; COP-coated vesicles; ER export; secretory pathway; dendrite; biological transport

\section{Introduction}

Long-term memory is thought to require long-lasting modifications of synapses as a result of an increase in synaptic transmission efficacy driven by correlations in the firing activity of presynaptic and postsynaptic neurons (Hebb, 1976). Some of these long-term modifications require new dendritic protein synthesis (Poirazi and Mel, 2001). One mechanism by which these newly synthesized proteins arrive at their dendritic targets is that they are synthesized within the soma and transported through the endoplasmic reticulum (ER) and Golgi compartments to be sorted for dendritic expression at the trans-Golgi network. However, this method of protein targeting is very inefficient for cargo destined to arrive at secondary and tertiary dendritic branches because of the great distances needed to be traveled (Steward and Worley, 2001). Therefore, neurons have developed a mechanism(s) to target specific mRNAs to dendritic localized translation machinery (Rao and Steward, 1993; Steward, 1994, 1995; Mohr et al., 1995; Crino et al., 1998; Eberwine et al., 2001; Steward and Worley, 2001). The discovery that some of these locally translated mRNAs encode for membrane proteins challenges the idea that membrane protein entry into the secretory pathway is restricted to the soma (Miyashiro et al., 1994; Johnston et al., 1996, 1999; Chen et al., 1997; Horton and

Received 0ct. 22, 2003; revised Feb. 17, 2004; accepted Feb. 19, 2004

M.A. is supported by grants from the Competitive Medical Research Fund of the University of Pittsburgh Medical Center and by the Edward Mallinckrodt Jr. Foundation. A.B. is supported by a postdoctoral fellowship from the Osteogenesis Imperfecta Foundation. K.N.F. is supported by the National Alliance for Research on Schizophrenia and Depression and National Institute of Mental Health Grant MH064372. We are grateful to Drs. Tamas Bartfai, William Balch, and Floyd Bloom for their interest, encouragement, and advice during discussions preceding this project. We thank Marga Behrens for her helpful input and discussions.

Correspondence should be addressed to Dr. Kenneth N. Fish, Department of Neuropharmacology, Mail Drop SV-229, The Scripps Research Institute, 10550 North Torrey Pines Road, La Jolla, CA 92037 . E-mail: kenfish@scripps.edu.

DOI:10.1523/JNEUROSCI.4775-03.2004

Copyright $\odot 2004$ Society for Neuroscience $\quad 0270-6474 / 04 / 243770-07 \$ 15.00 / 0$
Ehlers, 2003) and suggests that dendrites contain machinery, such as rough ER (RER) and Golgi, that would allow translation of the $\mathrm{mR}$ NAs and glycosylation of the protein products.

In support of the existence of a dendritic secretory pathway, translation and translocon machinery components have been detected in dendritic shafts and spines as far as dendritic branch points in several neuronal types (Steward and Banker, 1992; Krijnse-Locker et al., 1995; Tiedge and Brosius, 1996; Gardiol et al., 1999; Pierce et al., 2000; Ma and Morris, 2002). There is also immunocytochemical evidence that components of a functional ER and ER-to-Golgi transport system are present in the dendrites of some cell types (De Camilli et al., 1986; Takei et al., 1992; Torre and Steward, 1992, 1996; Villa et al., 1992; Lowenstein et al., 1994; Krijnse-Locker et al., 1995; Gardiol et al., 1999; Pierce et al., 2000, 2001; Ma and Morris, 2002; Horton and Ehlers, 2003).

To study dendritic ER protein sorting and to determine the specific location and extent of ER export in dendrites, we developed an in vitro primary hippocampal neuron system that enables us to manipulate and analyze localized ER export. This in vitro system capitalizes on the role of the soluble GTPase Sar1 in controlling ER export site formation and COPII (coat protein complex II) assembly (Aridor et al., 2001). Using our synchronized ER export assay, we show here that ER export sites are assembled regularly throughout the entire dendritic arbor and that activation of metabotropic glutamate receptors leads to the enhanced recruitment of the NMDA receptor subunit NR1 to remodeled ER export sites. We propose that regulation of receptor assembly and export from the ER in dendrites plays an important role in modulating receptor surface expression and neuronal function.

\section{Materials and Methods}

Antibodies and recombinant protein. Antibodies to mammalian Sar1, mammalian Sec23, and mammalian Sec13 were prepared as described 
previously (Plutner et al., 1992; Kuge et al., 1994; Aridor et al., 1995; Rowe et al., 1996). Recombinant wild-type Sar1, Sar1 [T39N], and Sar 1 [H79G] mutants were expressed and purified as described previously (Kuge et al., 1994; Aridor et al., 1995; Rowe and Balch, 1995; Rowe et al., 1996). Recombinant dynamin 1 was kindly provided by Dr. H. Damke (The Scripps Research Institute).

Dissociated cultures. Neurons were cultured at low density from embryonic day 18 Sprague Dawley rats essentially as described previously (Goslin and Banker, 1991). Cells were plated onto poly-L-lysine-coated glass coverslips at a density of 5400 cells $/ \mathrm{cm}^{2}$ to achieve low-density cultures, which were required for visualization of ER export sites. Most neurons, $>90 \%$, developed the characteristic mature morphology of spiny neurons between 16 and 21 days in vitro (DIV). As a measure of culture maturity in experiments using 21 DIV neurons, we confirmed that the majority of the cells in the culture had a relatively high density of dendritic spines (0.75-1.5 punctae per micrometer of dendrite) using rhodamine-phalloidin.

ER export assay. Cultures were rinsed with PBS, followed by permeabilization with $25 \mu \mathrm{g} / \mathrm{ml}$ digitonin at room temperature for $5 \mathrm{~min}$ and then washed three times with KHM buffer (in mM: 110 KOAc, 20 HEPES, pH 7.4, and $2 \mathrm{MgOAc}$ ) as described previously (Plutner et al., 1992). Cells were prepared and incubated with the indicated components as described. At the end of the incubations, the cells were fixed with $2 \%$ formaldehyde in PBS, and preparation for immunofluorescence (IF) microscopy was performed as described previously (Plutner et al., 1992).

Microscopy and export site quantification. Images were collected on an Olympus Optical (Melville, NY) FV500 confocal microscope or an Olympus Optical IX-70 microscope equipped with an ORCA-II digital camera (Hamamatsu, Bridgewater, NJ) and a Ludl motorized XYZ stage (LEP, Hawthorne, NY). Data was deconvolved using the Agard/Sadat inverse matrix algorithm.

To quantify vesicle formation within neurons, five sequential confocal slices taken $0.1 \mu \mathrm{m}$ apart through the midplane of the dendrites were collected with fixed laser illumination and pinhole size using a $60 \times, 1.4$ numerical aperture plan apochromat objective on an Olympus Optical FV500 confocal. Using ISee Imaging Systems (Raleigh, NC) software, three-dimensional (3D) images were constructed of each dendrite. The reconstructed images were then delineated, and the threshold was set. The number, size, and signal intensity within each vesicle was then measured. No significant difference was detectable in the grayscale values for each voxel within the vesicles in any of the cells or any of the conditions. Thus, our quantification is restricted to the number of the vesicles within dendrites, which were measured automatically, on the basis of threshold segmentation. Using these experimental parameters, the vesicles of dendrites from five neurons per condition were quantified.

\section{Results}

The COPII coat is assembled at distal dendritic sites in vitro

To test the hypothesis that ER export sites are assembled throughout the dendritic arbor, we developed an in vitro system that enabled us to manipulate ER export in primary neurons. In this assay, access to the ER membrane is accomplished by the exposure of primary neuron cultures to digitonin (Plutner et al., 1992; Davidson and Balch, 1993; Aridor et al., 1998, 2001), which selectively permeabilizes the plasma membrane (PM), exposing functionally and morphologically intact ER. In this system, we also use a transdominant form of Sar 1 protein that is locked in the active GTP-bound state, Sar1-GTP (Sar1 [H79G]), which leads to the stable recruitment of COPII from the cytosol to the membrane at ER export sites. Because the COPII coat complexes are recruited sequentially, Sar 1 activation leads to the recruitment of Sec23/24, followed by the recruitment of Sec13/31; membranebound Sec13/31 represents fully assembled ER export sites. Therefore, we analyzed the localization of membrane-bound Sec13/31 complexes in 21 DIV hippocampal neurons. The neurons were permeabilized, rinsed, and incubated with rat liver cytosol (RLC) (a source of COPII components Sec23/24 and
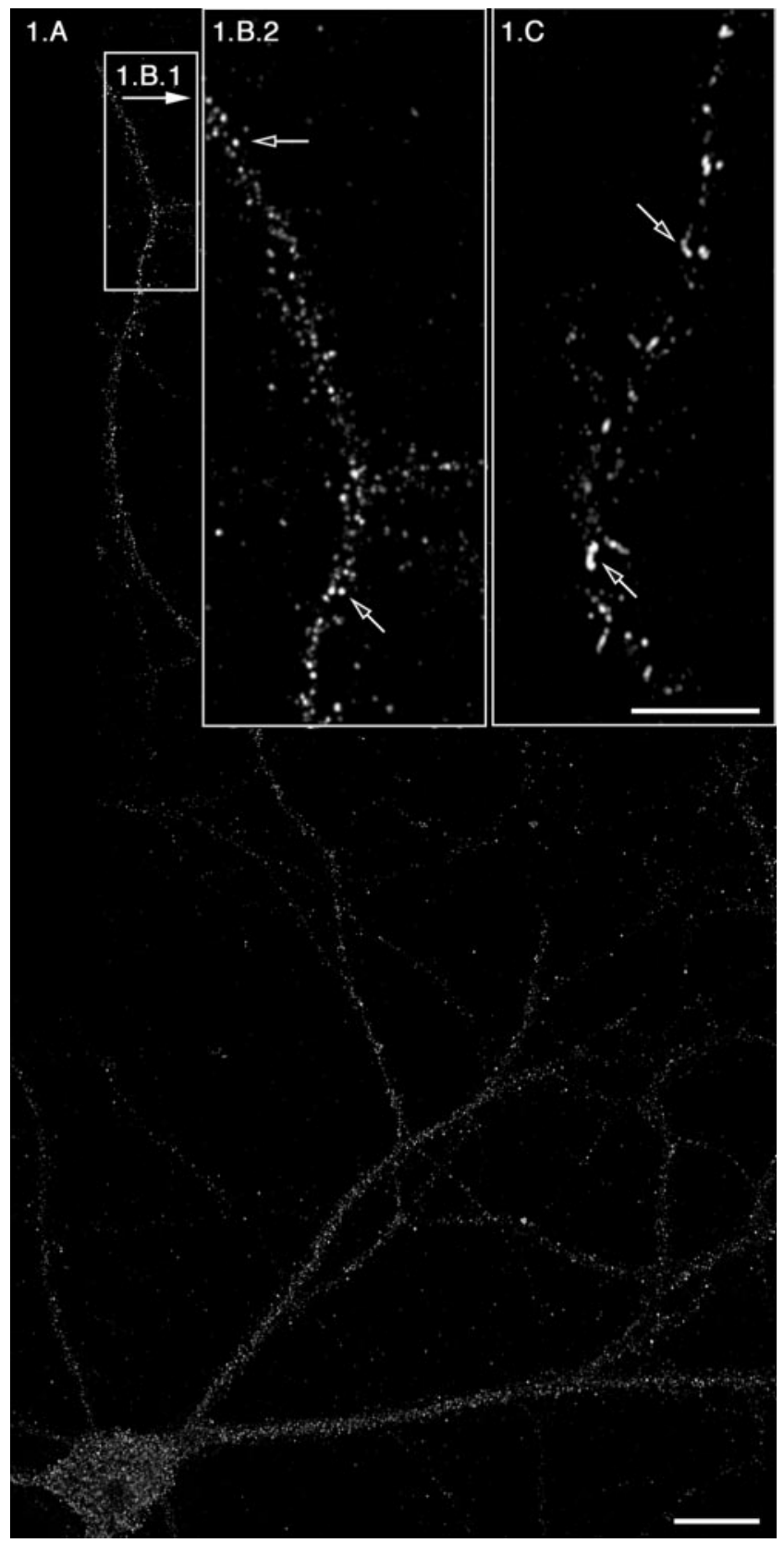

Figure 1. ER export occurs efficiently and regularly at distances $>350 \mu \mathrm{m}$ from the soma. Rat hippocampal neurons were rinsed with PBS, permeabilized, and washed as described in Materials and Methods. Cells were then incubated in the presence of RLC and $5 \mu \mathrm{g}$ of Sar1-GTP $(A, B 1, B 2)$ or with Sar1-GTP only ( $C$. At the end of the incubations, the distribution of $\operatorname{Sec} 13(A$, $B 1, B 2$ ) and $\operatorname{Sar} 1$ ( $C$ ) was determined using IF microscopy. The open arrows in $B 2$ denote punctate VTCs. The open arrows in C denote ER membrane tubules coated with Sar1. B2 and C are higher magnifications of tertiary dendrites that are $>350 \mu \mathrm{m}$ away from soma. Scale bars: $A$, $20 \mu \mathrm{m}$; (in C) $B 2, C, 10 \mu \mathrm{m}$.

Sec13/31) and recombinant Sar1-GTP (Fig. 1A,B1,B2). Because rinsing after permeabilization removes the endogenous cytosolic COPII machinery, in the presence of Sar1-GTP, but absence of RLC, COPII coat proteins are not recruited to the ER membrane (data not shown). The addition of Sar1-GTP and RLC led to a dramatic recruitment of Sec13, as observed by the characteristic punctate staining, which represents newly assembled COPII carriers. These structures were identified previously as vesicular tubular clusters (VTCs) that form during COPII budding (Saraste and Svensson, 1991; Bannykh et al., 1996). Because the activated 
Sar1-GTP inhibits COPII uncoating, these COPII-coated vesicles fail to form mature VTCs and appear attached to each other as elongated "beads on a string," structures that are located at ER export sites (Bannykh et al., 1996). Sar1 under these conditions is localized to these elongated sites, which contain recruited punctated Sec13 (data not shown). Notably, Sec13 recruitment was blocked by the addition of the protein kinase inhibitor H89, which inhibits Sar1 recruitment, COPII assembly, and ER export (Muniz et al., 1996, 1997; Aridor and Balch, 2000; Leemhuis et al., 2002) (Fig. 2 and data not shown). Importantly, the recruitment of Sec13 is not restricted to the soma but is observed throughout the dendrites: fully assembled COPII-coated sites are visible $>350 \mu \mathrm{m}$ away from the soma in tertiary dendritic branches (Fig. $1 A, B 1, B 2)$. Thus, COPII assembly is observed throughout the dendritic tree.

Sar1-GTP triggers the formation of ER membrane tubules in the soma and dendrites of rat hippocampal neurons-sites of ER export

We demonstrated previously that Sar 1 initiates cargo recognition in vitro by incubating permeabilized normal rat kidney (NRK) cells with activated Sar1 in the absence of COPII components, which results in the formation of elongated tubular export sites (Aridor et al., 2001). These sites were shown to selectively accumulate biosynthetic cargo and were primarily devoid of ER resident proteins. We used this methodology to stabilize elongated ER export sites for analysis in neurons, thus providing us with a refined picture of ER export site formation throughout the entire neuron. Hippocampal neurons were permeabilized, washed, and incubated in vitro as described above. After a $45 \mathrm{~min}$ incubation, Sarl protein distribution was examined by IF microscopy. When permeabilized neurons were incubated in the absence of COPII coat machinery, residual punctate Sarl staining was observed (Fig. 2A1). In contrast, when cells were incubated in the presence of Sar1-GTP, long ER tubular domains formed in the soma (Fig. 2A2) and proximal dendrites (Fig. 2 B1,B2). Tubular domains also formed in secondary dendritic branches but at a lower frequency (Fig. 2 B1,B3). Although tubular domains were not easily detected in tertiary branches, Sar1 was still heavily recruited (Fig. $2 B 1, B 4)$. These structures extended up to $10 \mu \mathrm{m}$ in the soma of neurons (Fig. $2 A 2$ and data not shown) and to $2-4 \mu \mathrm{m}$ in dendrites (Fig. 2B1-B4 and data not shown). Importantly, Sar1 recruitment was clearly visible $>350 \mu \mathrm{m}$ away from the soma in tertiary dendritic branches (Fig. $1 C$ ). The elongated tubular export sites generated under these conditions are exaggerated structures formed as a result of the artificially prolonged lifetime of an otherwise transient intermediate and represent an early intermediate in export from the ER (Aridor et al., 2001). Because differences in the amount of mRNAs and secretory pathway components have been described when analyzed in different neurons, we also examined the formation of ER export sites in permeabilized cortical neurons (supplemental Fig. 1, available at www. jneurosci.org). Sar1 recruitment and ER membrane tubule formation (supplemental Fig. $1 A$, Sar1 staining) and assembly of COPII carriers marked by Sec13 staining (supplemental Fig. $1 B$ ) were similar to that in hippocampal cells. The controls described above for our studies on hippocampal neurons were also performed here in cortical neurons, yielding similar results (data not shown).

We next analyzed the requirement of Sarl activation in the formation of the ER tubular domains and the specificity of this reaction in neurons. Tubular domains were not observed when permeabilized neurons were incubated on ice in the presence of Sar1-GTP (data not shown), nor when incubations were per-
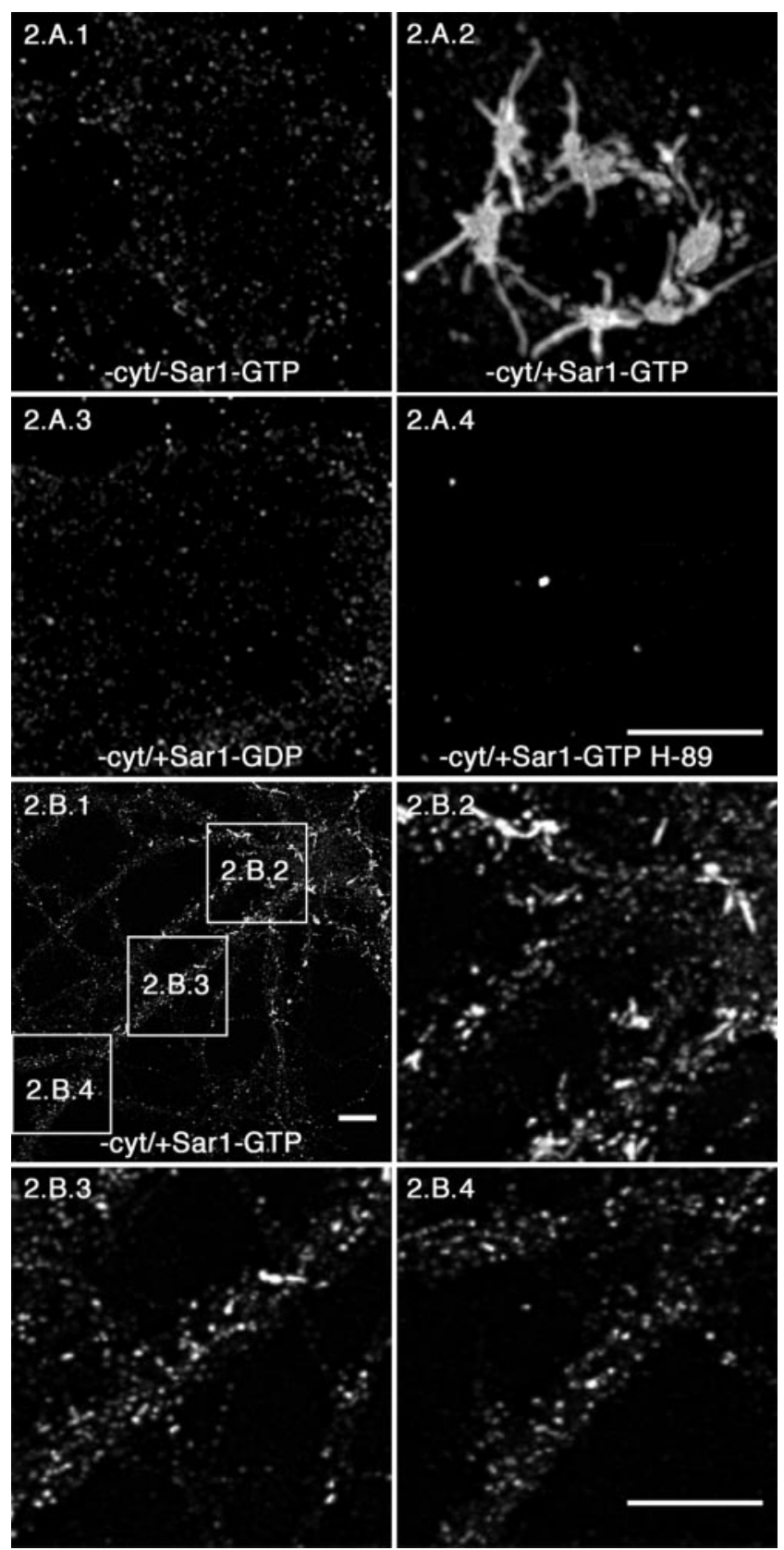

Figure 2. Sar1-GTP triggers the formation of ER membrane tubules in rat hippocampal neurons-sites of ER export. Permeabilized rat hippocampal neurons $(A)$ were incubated in reaction buffer only $(A 1)$ or with Sar1-GTP only $(A 2)$ as described in Materials and Methods. The neurons in $A 3$ were incubated with Sar1-GDP to determine whether Sar1 activation was required for ER tubule formation. Cultures represented in $A 4$ were incubated in the presence of Sar1-GTP and the isoquinoline protein kinase inhibitor H89. The distribution of Sar1 was determined using IF microscopy. All images in $A$ are $3 D$ reconstructions from confocal image stacks of Sar1 staining in the cell body. $B$, Rat hippocampal neurons were incubated in the presence of Sar1-GTP only and prepared for IF microscopy using antibody directed toward Sar1 as described above. B2-B4 are higher magnifications of proximal, secondary, and tertiary dendritic branches, respectively. 3 D reconstructions were made from confocal stacks taken at the level of the dendrites (1-2.5 $\mu \mathrm{m}$ above the cover glass). Arrows denote Sar-1-coated ER membrane tubules. Scale bars, $10 \mu \mathrm{m}$. cyt, Cytosol.

formed in the presence of the inactive form of Sar1 (Sar1-GDP; Sar1 [T39N] mutation) (Fig. 2A3). In addition, tubules were not observed when neurons were incubated in the presence of recombinant dynamin 1, which can tubulate the PM and synthetic liposomes in vitro (data not shown) (Takei et al., 1998). Further- 
more, there was an almost complete loss of Sar1 staining when cells were incubated in the presence of Sar1-GTP and the protein kinase inhibitor H89 (Fig. 2A4).

Under the same conditions used for our neuronal assay, incubation of permeabilized NRK cells in the presence of Sar1-GTP alone resulted in the formation of ER-derived tubular domains marked by Sar1 staining (supplemental Fig. 1C). NRK ER tubule formation under these experimental conditions was similar to our previously published results with these cells (Aridor et al., 2001). Together, these data establish that tubule formation in neurons requires Sarl activation on ER membranes.

\section{Sar1 generates tubules that are functional intermediates in ER export in vitro}

If Sar1-mediated tubule formation does in fact represent a functional early intermediate in ER export, then Sarl tubules should be able to mediate later events in ER-to-Golgi transport. To visualize the completion of the ER export step in cells after Sar1 activation and tubule formation, we examined the fate of Sar1dependent tubules morphologically after the addition of RLC to permeabilized cells using a two-stage assay. In stage 1, permeabilized neurons were incubated with Sar1-GTP to form extended tubules. Neurons were then thoroughly rinsed, and RLC containing COPII components Sec23/24 and Sec13/31 was subsequently added (stage 2). Neurons were incubated in stage 2 for $10 \mathrm{~min}$ at $37^{\circ} \mathrm{C}$ to promote ER export. The recruitment of COPII components under these conditions was examined by confocal microscopy using an antibody directed toward Sec23 (supplemental Fig. 2). Addition of RLC in the second stage resulted in heavy recruitment of Sec23/24 (supplemental Fig. 2A) and Sec 13/31 (data not shown) in both the soma (supplemental Fig. 2A1) and dendrite (supplemental Fig. 2A2). When Sar1-GTP was left out of the first incubation, almost no COPII components were recruited (supplemental Fig. $2 B, B 1$ and data not shown). These results demonstrate that Sar1-GTP-induced tubules can efficiently be converted to VTCs.

\section{COPII is assembled on export sites in distal dendritic branches in vivo}

Previous studies have localized components of the translocon machinery to dendritic arbors (Tiedge and Brosius, 1996; Pierce et al., 2000, 2001). To corroborate our in vitro findings, we compared the localization of endogenous Sarl and Sec13 to that of the RER translocon $(\operatorname{Sec} 61 \alpha)$ machinery in primary neurons, thus comparing protein entry points into the ER (Sec61 $\alpha$ ) with sites of exit (Sar1 and Sec13). The localization of Sec61 $\alpha$ (supplemental Fig. 3A, green), Sar1 (supplemental Fig. 3B, green), and Sec13 (supplemental Fig. $3 C$, green) in relation to dendritic spines (phalloidin staining, red) at tertiary dendritic branch points is presented in supplemental Figure 3. Sec61 $\alpha$, Sar1, and Sec13 were clearly visible in medial-tertiary branches of mature neurons. These results support our in vitro observations that COPII assembly occurs throughout the entire dendritic arbor. Therefore, local translation of mRNA coding for membrane proteins, protein translocation into the ER, and ER export can take place in the dendrite independently of the soma.

\section{Formation of distal ER export sites is regulated by dendritic maturation}

To determine whether a direct correlation exists between local ER export site formation in dendrites and neuronal maturation, we quantified the density of ER export sites in somatic, proximal, secondary, and tertiary dendritic branches in both maturing neu-

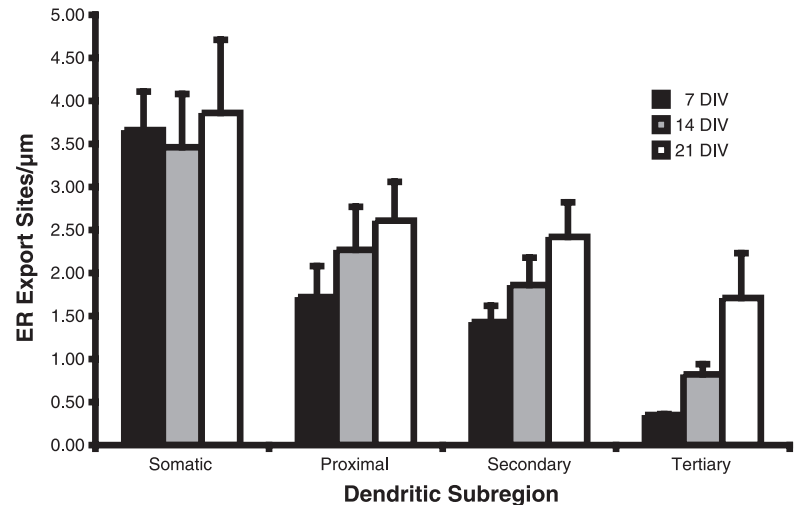

Figure 3. Quantification of ER export sites in hippocampal neurons. Using our two-stage assay (supplemental Fig. 2, available at www.jneurosci.org), Sec23 vesicles were quantified in neurons at the designated DIV. A modification of the "centrifugal method" of dendritic branch ordering was used to describe the density of dendritic arborizations (Uylings and van Pelt, 2002). Our characterization was such that the dendrites emerging from the cell soma are called somatic for one soma diameter distance and then primary up to the point of bifurcation into second-order branches. Therefore, first branches are called secondary and so on, with increasing order until the tips are reached. For these experiments, the vesicles of dendrites from a minimum of five neurons, from at least three different experiments, at each time point were quantified. Values equal mean \pm SEM.

rons (7 and 14 DIV) and mature neurons (21 DIV). Because Sar1-GTP is capable of producing several morphologically different ER export sites (tubules of different length), quantification was performed on vesicles formed in the two-stage assay (described above) that stained positive for Sec23. These vesicles are extremely uniform and are more directly related to the number of ER export sites than when formed by the coincubation of Sar1GTP and RLC, which can potentially result in multiple vesicles arising from a single export site. As represented in Figure 3, neurons at 7 DIV already present ER export sites in distal processes, albeit at low density relative to their distribution in somatic dendritic branches. As the neuron matures, the density of ER export sites within somatic dendrites is essentially unchanged. In contrast, the density of ER export sites in the proximal, secondary, and in particular tertiary dendritic branches is dramatically increased. In tertiary dendritic branches, the density of ER export sites nearly quadrupled between 7 and 21 DIV. The selective increase in dendritic ER export site density in tertiary dendritic branches supports our hypothesis that these export sites are required to support dendritic postsynaptic activities.

\section{Sar1 export sites recruit dendritic-specific cargo}

To determine whether Sar1 ER export sites were used by dendritic specific-cargo, we analyzed the ability of Sar1-induced export sites to capture NR1, a subunit of the NMDA receptor (Nakanishi, 1992) whose mRNA is selectively targeted and transported to dendrites (Steward and Schuman, 2001). The NMDA receptor includes NR1 and NR2 subunits that are assembled in the ER (Wenthold et al., 2003). NR1 mRNA translation was induced by 3,5dihydroxyphenylglycine (DHPG), a highly selective agonist for group I metabotropic glutamate receptors (Schoepp, 1994; Gereau and Conn, 1995; Kacharmina et al., 2000). The ability to physiologically induce endogenous expression of this receptor subunit provided us with a unique opportunity to monitor localized cargo capture in dendritic ER export sites (Kacharmina et al., 2000). DHPG induction of NR1 synthesis followed by Sar1-GTP tubule formation resulted in the concentration of newly synthesized NR1 in Sar1-GTP ER tubules. This is seen in the colocaliza- 


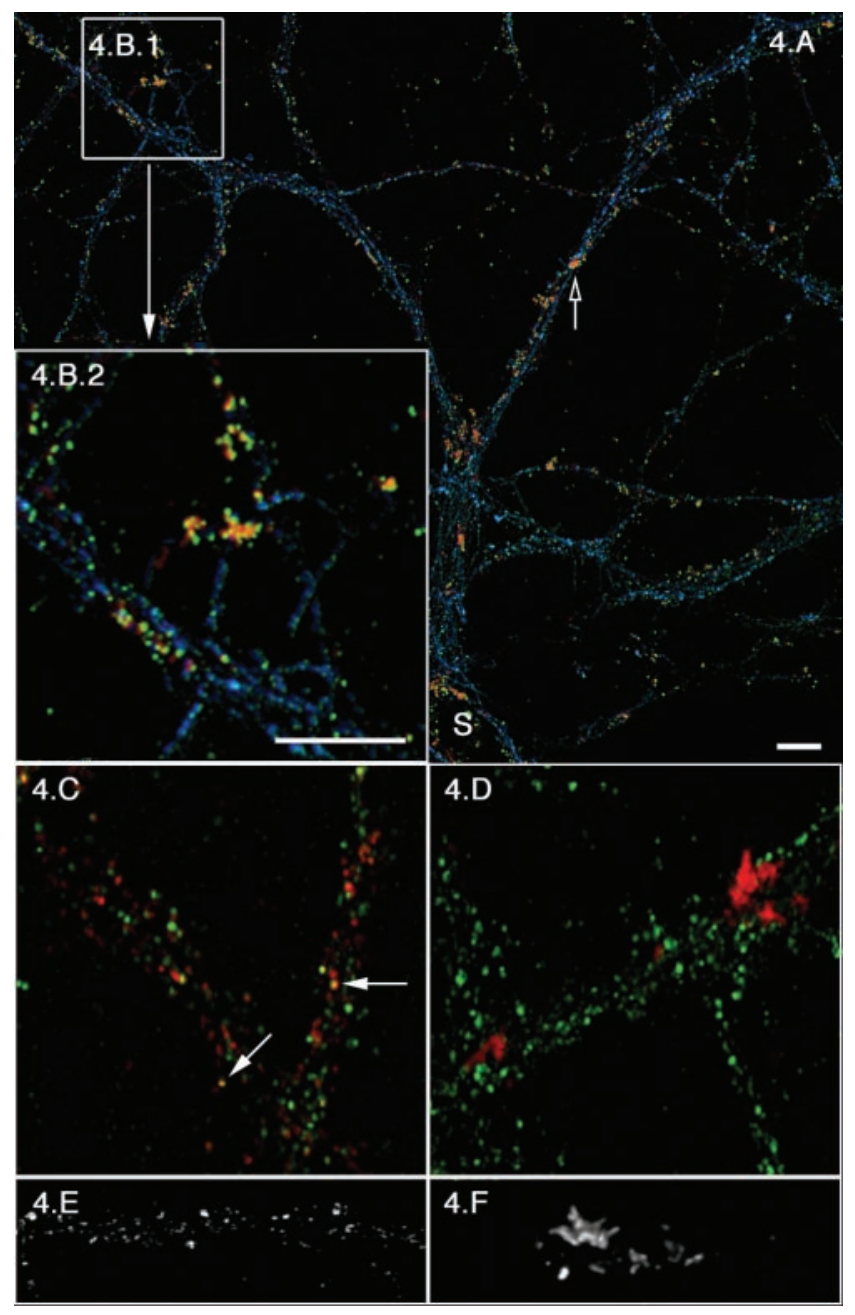

Figure 4. DHPG treatment results in Sar1 tubule reorganization and the selective concentration of NR1 in ER tubule domains. Control $(C, E)$ or DHPG (15 min at a $100 \mu \mathrm{m}$ final concentration; $A, B, D, F)$-treated 21 DIV hippocampal neurons were permeabilized, rinsed, incubated with $5 \mu \mathrm{g}$ of Sar1-GTP, and fixed as described in Figure 1. $A$ is a lower-magnification image of NR1 (green), Sar1 (red), and phalloidin (blue). Open arrows denote hot spots of ER export and Sar1/NR1 colocalization (yellow). B2, which is a $3 \times$ magnification of $B 1$, clearly represents the extent of Sar1/NR1 colocalization. C represents a similar region in a control culture (NR1, green Sar1, red; filled arrows denote sites of colocalization). Specificity of Sar1/NR1 colocalization was controlled for in $D$, in which there is no colocalization of $\alpha$ subunit of CaMKII (green) with Sar1 (red) in DHPG-treated neurons. $A-D$ are extended focus images compiled from small confocal stacks (sections are 0.1-0.2 $\mu \mathrm{m}$ apart) to avoid false colocalization. $E$ (control) and $F$ (DHPG treated) are 3D reconstructions of Sar1 staining in tertiary dendritic branches. Images were made from confocal stacks with consecutive sections taken $0.1 \mu \mathrm{m}$ apart. S, Soma. Scale bars, $10 \mu \mathrm{m}$.

tion of assembled ER export sites, marked by Sar1 staining (red), with the NR1 subunit (green) in the soma and throughout dendrites (Fig. 4). A more detailed view of the association of NR1 (green) with Sar1 (red)-coated export sites in a tertiary dendrite (Fig. 4 B2) clearly demonstrates that the export sites characterized in distal dendritic branches can capture dendritic cargo and are therefore functional. In addition, there is a clear increase in Sar 1/ NR1 colocalization in DHPG-treated neurons over untreated cultures (Fig. 4, compare B2,C). As a control, we analyzed the distribution of the $\alpha$ subunit of calcium-calmodulin-dependent protein kinase II (CaMKII) (green), a cytosolic protein whose mRNA is present at high concentrations throughout dendrites (Steward, 1997) (Fig. 4D). The results of this experiment demonstrate the specificity of Sar1/NR1 colocalization. Analysis of ER export sites in a tertiary branch by high-magnification 3D reconstruction confocal microscopy shows that multiple elongated tubules emanate from defined foci in DHPG-treated cultures (Fig. 4F). These tubular domains, which now resemble the Sar1coated tubules formed in the soma (Fig. 2A2), are markedly different from the punctated and short tubular morphology observed in the absence of DHPG (Fig. 4, compare B2,D,F with $C, E$ ). Although the mechanism(s) by which specific local dendritic protein synthesis can lead to altered export site morphology is unknown, it suggests that receptor expression at the dendrite membrane surface can be regulated and adjusted during export from localized ER export sites.

\section{Discussion}

The first step in delivery of newly synthesized receptors and ion channels to the PM is their cotranslational insertion into the ER membrane. In the ER, these proteins undergo regulated folding, which controls protein complex assembly, cargo capture, and export by masking ER retention signals or exposing export signals. Protein processing and sorting in the ER may therefore play a key role in regulating surface expression. In neurons, this role may need to be exploited locally in dendrites because the transport of dendritic cargo proteins from the cell soma and somatic dendrite to secondary and tertiary dendritic branches can be a slow and complicated task hindered by their elongated and asymmetrical morphology. To investigate whether localized ER export sites exist throughout the entire dendritic tree to facilitate and regulate secretory pathway protein delivery to specific sites in the $\mathrm{PM}$, we reconstructed the process of ER export in primary dissociated hippocampal neurons. Our experimental results demonstrate that biosynthetic cargo continuously exits the ER in mature neurons from the soma to the most distal dendritic branches. Importantly, the number of ER export sites in distal dendritic branches increases as neurons mature and establish synaptic connections. Furthermore, dendritic ER export sites are remodeled in response to a physiological increase in dendritic ER cargo load. From these results, we conclude that localized ER export is used throughout the dendritic arbor to regulate surface protein expression.

Characterization of ER export in dendrites was performed using in vitro assays that separate this event into discrete steps. The first step was visualized when digitonin permeabilized neurons were incubated in the presence of Sar1-GTP alone, which resulted in the formation of tubular ER-derived domains in somatic, secondary, and tertiary dendritic branches. The Sarl tubules generated in the soma and proximal dendrites are elongated membranes, whereas the ER export domains formed in medial and distal dendritic regions are short tubular and punctated structures. The specificity of these morphological changes as an activated Sar1-dependent event is supported by the results that ER tubule formation is blocked by incubation with the dominant-negative Sar1-GDP (Fig. 2). COPII assembly in dendrites, the next step in ER cargo export, was demonstrated by the efficient recruitment of Sec13 (Fig. 1). Sec13 recruitment was dependent on Sar1 activation and was blocked by the addition of the kinase inhibitor H89, which prevents Sar1 recruitment and activation. Using a two-stage assay, we reconstructed the transition from Sar 1 tubule to COPII-coated VTCs in vitro and quantitated the number of dendritic ER export sites in developing and mature neurons. These experiments showed that, as neurons mature, the density of ER export sites within the cell body and somatic dendrites remain essentially unchanged but increases in proximal, secondary, and tertiary branches, in which they peaked at the same time as dendritic spine density (Fig. 3). The dramatic 
increase in dendritic ER export site density in tertiary branches of 21 DIV neurons suggest that there is a direct relationship between the formation of stable synaptic connections, a process that requires biosynthesis and protein trafficking to the cell surface, and ER export site density.

The results published here are supported by the recent paper by Horton and Ehlers (2003) in which they demonstrated that an exogenously expressed viral reporter protein, VSV-G (vesicular stomatitis virus glycoprotein-G), could exit the ER in both the soma and dendrites. Using our ER export assay and exogenously expressed VSV-G, we found similar results, which we presented at the 2001 Annual Society for Neurosciences meeting (Fish et al., 2001). Horton and Ehler also showed that some of the dendritically targeted VSV-G was transported to local dendritic Golgi compartments after exiting the ER. Interestingly, they found that the amount of dendritic Golgi increased as neurons matured, peaking at 12 DIV when cells were undergoing extensive process outgrowth and synaptogenesis. Thus, the incremental increases in dendritic ER export sites documented here correlates well with the age-dependent increase of dendritic Golgi outposts documented by Horton and Ehler. The role of export from the ER in a possible de novo local formation of Golgi compartments is a debated issue that will be addressed in future studies. However, the results of both Horton and Ehlers and ours suggest that the early secretory pathway is remodeled during neuronal development to support local biosynthetic protein transport in dendrites.

To determine the physiological significance of dendritic ER export sites, we assessed their ability to capture biosynthetic cargo. For these experiments, we used DHPG to induce local dendritic protein synthesis before Sar1-GTP tubule formation. Because NMDA receptors are key signaling components of the brain and are present as one of the major dendritic membrane proteins in proximal and distal dendrites of hippocampal and cortical neurons, we wanted to determine whether newly synthesized NR1 colocalized with ER tubules (Fig. 4). NR1 was an ideal membrane protein to study in these experiments because the NR1 mRNA is selectively targeted and transported to dendrites (Steward and Schuman, 2001). A surprising and important result from these experiments is that the induction of specific local dendritic protein synthesis leads to a morphological change in tertiary ER export sites, which may be a specific response to local cargo load. This hypothesis is supported by experiments we performed previously in NRK cells using VSV-G as an ER cargo reporter (Aridor et al., 1999). In these experiments, VSV-G appeared to modulate the activity of the ER export machinery. Moreover, recent in vitro analysis of vesicle formation using Sec23/24 proteins in which selected cargo recognition motif was mutated leading to a selective deficiency in specific cargo capture led to the formation of COPII vesicles with reduced size (Miller et al., 2003). Our observations that the upregulation of endogenous dendritic protein synthesis can modulate the morphological appearance of ER export sites suggests that such regulation may play an important physiological role: receptor expression at the dendrite PM can be regulated and adjusted during export from localized ER export sites.

The discovery that endogenous proteins exit the ER in distal dendritic branches and that, in mature neurons, export occurs in this subdendritic location sheds light on the mechanism by which the requirement for newly synthesized membrane proteins in the most distal regions of dendrites is fulfilled in long-term memory (Johnston et al., 1996, 1999; Chen et al., 1997). It is important to point out that, although essential components for translocation have been detected in distal dendritic branches here (supplemental Fig. 3, available at www.jneurosci.org) and previously (Pierce et al., 2000), our finding that cargo leaves the ER does not require that all proteins exiting the ER in dendrites entered the ER in these branches. The smooth extended tubular domains of the smooth ER, which participate in vesicle-mediated export of cargo, form a continuous reticulum in dendrites (Martone et al., 1993; Krijnse-Locker et al., 1995; Cooney et al., 2002). Therefore, it is possible for a protein to be inserted into the membrane of the ER in the soma or in proximal and secondary dendrites and exit the ER in a distal branch. Nonetheless, our finding that NR1 was mostly captured exiting the ER at sites of DHPG-induced morphological changes in ER tubules supports the hypothesis that the local ER machinery is controlling the subdendritic targeting of NR1. Another hypothesis may be that the location of ER export is being influenced by the subdendritic location of mRNA. During translational stimulation, an increase in local ER cargo load occurs, which results in the morphological changes in ER tubules. Future work will evaluate the relationship between where proteins enter the ER to where they exit.

It is well documented that complex regulation is exerted on the assembly and expression of NMDA receptors. For example, the C-terminal splice variant of NR1, C1, contains an RxR (retention/retrieval) type ER retention motif, which is disabled by protein phosphorylation. NR1-C2, which contains a PDZ (postsynaptic density-95/Discs large/zona occludens-1) binding motif, can assemble with NR1-C1 and mask the RxR motif, promoting ER export. Moreover, activity-dependant alternative splicing produces the NR1-C2' variant, which contains a divaline motif that facilitates the interactions of NR1 with COPII and ER export, and the ER export of NR2 is dependent on NR1 (Mu et al., 2003). Therefore, the regulation of receptor complex assembly is used to control the trafficking of NMDAR from the ER to the cell surface. We now suggest that reciprocal regulation is exerted on the COPII export machinery, whereby the physiological upregulation of NR1 leads to specific remodeling of dendritic ER export sites. Collectively, these results suggest that surface expression of receptors may be tightly and locally regulated in the dendritic ER. The neuronal in vitro reconstitution assay developed here will enable future studies to address the molecular basis for local protein assembly and export from the ER and its role in modulating dendrite functions.

\section{References}

Aridor M, Balch WE (2000) Kinase signaling initiates coat complex II (COPII) recruitment and export from the mammalian endoplasmic reticulum. J Biol Chem 275:35673-35676.

Aridor M, Bannykh SI, Rowe T, Balch WE (1995) Sequential coupling between COPII and COPI vesicle coats in endoplasmic reticulum to Golgi transport. J Cell Biol 131:875-893.

Aridor M, Weissman J, Bannykh S, Nuoffer C, Balch WE (1998) Cargo selection by the COPII budding machinery during export from the ER. J Cell Biol 141:61-70.

Aridor M, Bannykh SI, Rowe T, Balch WE (1999) Cargo can modulate COPII vesicle formation from the endoplasmic reticulum. J Biol Chem 274:4389-4399.

Aridor M, Fish KN, Bannykh S, Weissman J, Roberts TH, LippincottSchwartz J, Balch WE (2001) The Sar1 GTPase coordinates biosynthetic cargo selection with endoplasmic reticulum export site assembly. J Cell Biol 152:213-229.

Bannykh SI, Rowe T, Balch WE (1996) The organization of endoplasmic reticulum export complexes. J Cell Biol 135:19-35.

Chen WR, Midtgaard J, Shepherd GM (1997) Forward and backward propagation of dendritic impulses and their synaptic control in mitral cells. Science 278:463-467.

Cooney JR, Hurlburt JL, Selig DK, Harris KM, Fiala JC (2002) Endosomal compartments serve multiple hippocampal dendritic spines from a wide- 
spread rather than a local store of recycling membrane. J Neurosci 22:2215-2224.

Crino P, Khodakhah K, Becker K, Ginsberg S, Hemby S, Eberwine J (1998) Presence and phosphorylation of transcription factors in developing dendrites. Proc Natl Acad Sci USA 95:2313-2318.

Davidson HW, Balch WE (1993) Differential inhibition of multiple vesicular transport steps between the endoplasmic reticulum and trans Golgi network. J Biol Chem 268:4216-4226.

De Camilli P, Moretti M, Donini SD, Walter U, Lohmann SM (1986) Heterogeneous distribution of the cAMP receptor protein $\mathrm{RII}$ in the nervous system: evidence for its intracellular accumulation on microtubules, microtubule-organizing centers, and in the area of the Golgi complex. J Cell Biol 103:189-203.

Eberwine J, Miyashiro K, Kacharmina JE, Job C (2001) Local translation of classes of mRNAs that are targeted to neuronal dendrites. Proc Natl Acad Sci USA 98:7080-7085.

Fish KN, Bloom FE, Bartfai T, Balch WE, Aridor M (2001) Morphological analyses of endoplasmic reticulum cargo export in neurons. Soc Neurosci Abstr 27:277.19.

Gardiol A, Racca C, Triller A (1999) Dendritic and postsynaptic protein synthetic machinery. J Neurosci 19:168-179.

Gereau IV RW, Conn PJ (1995) Roles of specific metabotropic glutamate receptor subtypes in regulation of hippocampal CA1 pyramidal cell excitability. J Neurophysiol 74:122-129.

Goslin K, Banker G (1991) Rat hippocampal neurons in low density culture. In: Culturing nerve cells (Banker G, Goslin K, eds), pp 251-281. Cambridge, MA: MIT.

Hebb DO (1976) Physiological learning theory. J Abnorm Child Psychol 4:309-314.

Horton AC, Ehlers MD (2003) Dual modes of endoplasmic reticulum-toGolgi transport in dendrites revealed by live-cell imaging. J Neurosci 23:6188-6199.

Johnston D, Magee JC, Colbert CM, Cristie BR (1996) Active properties of neuronal dendrites. Annu Rev Neurosci 19:165-186.

Johnston D, Hoffman DA, Colbert CM, Magee JC (1999) Regulation of back-propagating action potentials in hippocampal neurons. Curr Opin Neurobiol 9:288-292.

Kacharmina JE, Job C, Crino P, Eberwine J (2000) Stimulation of glutamate receptor protein synthesis and membrane insertion within isolated neuronal dendrites. Proc Natl Acad Sci USA 97:11545-11550.

Krijnse-Locker J, Parton RG, Fuller SD, Griffiths G, Dotti CG (1995) The organization of the endoplasmic reticulum and the intermediate compartment in cultured rat hippocampal neurons. Mol Biol Cell 6:1315-1332.

Kuge O, Dascher C, Orci L, Rowe T, Amherdt M, Plutner H, Ravazzola M, Tanigawa G, Rothman JE, Balch WE (1994) Sarl promotes vesicle budding from the endoplasmic reticulum but not Golgi compartments. J Cell Biol 125:51-65.

Leemhuis J, Boutillier S, Schmidt G, Meyer DK (2002) The protein kinase A inhibitor H89 acts on cell morphology by inhibiting Rho kinase. J Pharmacol Exp Ther 300:1000-1007.

Lowenstein PR, Morrison EE, Bain D, Shering AF, Banting G, Douglas P, Castro MG (1994) Polarized distribution of the trans-Golgi network marker TGN38 during the in vitro development of neocortical neurons: effects of nocodazole and brefeldin A. Eur J Neurosci 6:1453-1465.

Ma D, Morris JF (2002) Protein synthetic machinery in the dendrites of the magnocellular neurosecretory neurons of wild-type Long-Evans and homozygous Brattleboro rats. J Chem Neuroanat 23:171-186.

Martone ME, Zhang Y, Simpliciano VM, Carragher BO, Ellisman MH (1993) Three-dimensional visualization of the smooth endoplasmic reticulum in Purkinje cell dendrites. J Neurosci 13:4636-4646.

Miller EA, Beilharz TH, Malkus PN, Lee MCS, Hamamoto S, Orci L, Schekman R (2003) Multiple cargo binding sites on the COPII subunit Sec24p ensure capture of diverse membrane proteins into transport vesicles. Cell 114:497-509.

Miyashiro K, Dichter M, Eberwine J (1994) On the nature and differential distribution of mRNAs in hippocampal neurites: implications for neuronal functioning. Proc Natl Acad Sci USA 91:10800-10804.

Mohr E, Morris JF, Richter D (1995) Differential subcellular mRNA targeting: deletion of a single nucleotide prevents the transport to axons but not to dendrites of rat hypothalamic magnocellular neurons. Proc Natl Acad Sci USA 92:4377-4381.

Mu Y, Otsuka T, Horton AC, Scott DB, Ehlers MD (2003) Activity- dependent mRNA splicing controls ER export and synaptic delivery of NMDA receptors. Neuron 40:581-594.

Muniz M, Alonso M, Hidalgo J, Velasco A (1996) A regulatory role for cAMP-dependent protein kinase in protein traffic along the exocytic route. J Biol Chem 271:30935-30941.

Muniz M, Martin ME, Hidalgo J, Velasco A (1997) Protein kinase A activity is required for the budding of constitutive transport vesicles from the trans-Golgi network. Proc Natl Acad Sci USA 94:14461-14466.

Nakanishi S (1992) Molecular diversity of glutamate receptors and implications for brain function. Science 258:597-603.

Pierce JP, van Leyen K, McCarthy JB (2000) Translocation machinery for synthesis of integral membrane and secretory proteins in dendritic spines. Nat Neurosci 3:311-313.

Pierce JP, Mayer T, McCarthy JB (2001) Evidence for a satellite secretory pathway in neuronal dendritic spines. Curr Biol 11:351-355.

Plutner H, Davidson HW, Saraste J, Balch WE (1992) Morphological analysis of protein transport from the ER to Golgi membranes in digitoninpermeabilized cells: role of the P58 containing compartment. J Cell Biol 119:1097-1116.

Poirazi P, Mel BW (2001) Impact of active dendrites and structural plasticity on the memory capacity of neural tissue. Neuron 29:779-796.

Rao A, Steward O (1993) Evaluation of RNAs present in synaptodendrosomes: dendritic, glial, and neuronal cell body contribution. J Neurochem 61:835-844.

Rowe T, Balch WE (1995) Expression and purification of mammalian Sarl. Methods Enzymol 257:49-53.

Rowe T, Aridor M, McCaffery JM, Plutner H, Nuoffer C, Balch WE (1996) COPII vesicles derived from mammalian endoplasmic reticulum microsomes recruit COPI. J Cell Biol 135:895-911.

Saraste J, Svensson K (1991) Distribution of the intermediate elements operating in ER to Golgi transport. J Cell Sci 100:415-430.

Schoepp DD, Goldsworthy J, Johnson BG, Salhoff CR, Baker SR (1994) 3,5dihydroxyphenylglycine is a highly selective agonist for phosphoinositidelinked metabotropic glutamate receptors in the rat hippocampus. J Neurochem 63:769-772.

Steward O (1994) Dendrites as compartments for macromolecular synthesis. Proc Natl Acad Sci USA 91:10766-10768.

Steward O (1995) Targeting of mRNAs to subsynaptic microdomains in dendrites. Curr Opin Neurobiol 5:55-61.

Steward O (1997) mRNA localization in neurons: a multipurpose mechanism? Neuron 18:9-12.

Steward O, Banker GA (1992) Getting the message from the gene to the synapse: sorting and intracellular transport of RNA in neurons. Trends Neurosci 15:180-186.

Steward O, Schuman EM (2001) Protein synthesis at synaptic sites on dendrites. Annu Rev Neurosci 24:299-325.

Steward O, Worley PF (2001) Selective targeting of newly synthesized Arc mRNA to active synapses requires NMDA receptor activation. Neuron 30:227-240.

Takei K, Stukenbrok H, Metcalf A, Mignery GA, Sudhof TC, Volpe P, De Camilli P (1992) $\mathrm{Ca}^{2+}$ stores in Purkinje neurons: endoplasmic reticulum subcompartments demonstrated by the heterogeneous distribution of the InsP3 receptor, $\mathrm{Ca}^{2+}$-ATPase, and calsequestrin. J Neurosci 12:489-505.

Takei K, Haucke V, Slepnev V, Farsad K, Salazar M, Chen H, De Camilli P (1998) Generation of coated intermediates of clathrin-mediated endocytosis on protein-free liposomes. Cell 94:131-141.

Tiedge H, Brosius J (1996) Translational machinery in dendrites of hippocampal neurons in culture. J Neurosci 16:7171-7181.

Torre ER, Steward O (1992) Demonstration of local protein synthesis within dendrites using a new cell culture system that permits the isolation of living axons and dendrites from their cell bodies. J Neurosci 12:762-772.

Torre ER, Steward O (1996) Protein synthesis within dendrites: glycosylation of newly synthesized proteins in dendrites of hippocampal neurons in culture. J Neurosci 16:5967-5978.

Uylings HB, van Pelt J (2002) Measures for quantifying dendritic arborizations. Network 13:397-414.

Villa A, Sharp AH, Racchetti G, Podini P, Bole DG, Dunn WA, Pozzan T, Snyder SH, Meldolesi J (1992) The endoplasmic reticulum of Purkinje neuron body and dendrites: molecular identity and specializations for $\mathrm{Ca}^{2+}$ transport. Neuroscience 49:467-477.

Wenthold RJ, Prybylowski K, Standley S, Sans N, Petralia RS (2003) Trafficking of nmda receptors. Annu Rev Pharmacol Toxicol 43:335-358. 